\title{
Conhecimento popular sobre plantas medicinais em comunidades rurais de mata atlântica - Itacaré, BA, Brasil ${ }^{1}$
}

\author{
Erika de Paula Pedro Pinto ${ }^{2,4}$, Maria Christina de Mello Amorozo ${ }^{3}$ e Antonio Furlan ${ }^{3}$
}

\author{
Recebido em 17/08/2004. Aceito em 17/04/2006
}

\begin{abstract}
RESUMO - (Conhecimento popular sobre plantas medicinais em comunidades rurais de Mata Atlântica - Itacaré, BA, Brasil). O presente trabalho teve por objetivo o levantamento etnobotânico sobre o conhecimento e uso de plantas medicinais em duas comunidades rurais (Marambaia e Camboinha), localizadas em uma Área de Proteção Ambiental, na Mata Atlântica do Sul da Bahia, Brasil. Estas comunidades têm usado plantas medicinais como uma importante atividade terapêutica, a qual permite a auto-suficiência da população rural em relação aos cuidados com a saúde. Os dados foram coletados através de entrevistas com 26 famílias (24\% do número total). As plantas medicinais coletadas (98 espécies) foram catalogadas, identificadas e depositadas no Herbário Rio Clarense (HRCB). Elas pertencem a 40 famílias, dentre as quais Lamiaceae foi a mais citada. A maioria destas espécies (78\%) é cultivada, comumente nos quintais, pelos moradores locais. A folha é a parte da planta mais usada nos preparos medicinais. As espécies com maior número de citações são Chenopodium ambrosioides L. (mastruz) e Lippia alba (Mill) N.E. Br. (erva-cidreira), também associadas ao maior número de usos terapêuticos. Comparam-se no presente trabalho os índices de concordância de uso e de diversidade obtidos com os de outros estudos realizados em Florestas Tropicais no Brasil.
\end{abstract}

Palavras-chave: plantas medicinais, etnobotânica, Mata Atlântica, práticas populares

\begin{abstract}
Folk knowledge about medicinal plants within rural communities in Atlantic Forest, Itacaré, Bahia State, Brazil). This study's goal was to caryy out an ethnobotanical survey focusing on the knowledge and use of medicinal plants within two rural communities (Marambaia and Camboinha), which are situated in an Environmental Protection Area in Atlantic Forest of Southern Bahia, Brazil. These communities use medicinal plants as an important therapeutic activity, which permits the rural inhabitants to be selfsufficient regarding health care. Data were collected through interviews with 26 families ( $24 \%$ of the total). The medicinal plants collected (98 species) were catalogued, identified and deposited at the Herbarium Rio Clarense (HRCB). They belong to 40 families so that Lamiaceae was the most cited. The majority of these species (78\%) are cultivated, usually in backyards by local inhabitants. The leaf is the most common part of the plant used in medicinal preparations. The species with the greatest number of citations were Chenopodium ambrosioides L. and Lippia alba (Mill) N.E. Br. These species are also associated with the highest number of therapeutic uses. Use agreement and diversity index from this survey were compared to other surveys conducted in Brazilian Tropical Forests.
\end{abstract}

Key words: medicinal plants, ethnobotany, Atlantic Forest, folk knowledge

\section{Introdução}

As populações humanas que ocupam florestas tropicais convivem com a grande diversidade destes ambientes e desenvolvem, cada qual à sua maneira, formas de explorá-los para sua sobrevivência. De seu repertório cultural, destaca-se o conhecimento sobre o uso de plantas para fins medicinais.

As práticas relacionadas ao uso popular de plantas medicinais são o que muitas comunidades têm como alternativa viável para o tratamento de doenças ou manutenção da saúde. Porém, sua continuidade pode ser ameaçada pela interferência de fatores externos à dinâmica social do grupo como, por exemplo: a) maior exposição das comunidades à sociedade envolvente e, conseqüentemente, às pressões econômicas e culturais externas (Amorozo \& Gély 1988; Amorozo 2002); b) maior facilidade de acesso aos serviços da medicina moderna (Nolan 1999; Lima et al. 2000; Amorozo 2002); c) deslocamento das pessoas de seus ambientes naturais para regiões urbanas, o que leva à perda do caráter utilitário do conhecimento popular acumulado há várias gerações e, conseqüentemente, ao seu desaparecimento (Valle 2002).

\footnotetext{
Bolsa de Iniciação Científica da primeira Autora

2 Instituto de Pesquisa Ambiental da Amazônia - SCLN 210, Bloco C sala 211, 70862-530 Brasília, DF, Brasil

3 Universidade Estadual Paulista "Julio de Mesquita Filho", Departamento de Botânica, IB, C. Postal 199, 13506-900 Rio Claro, SP, Brasil

4 Autor para correspondência: erika@ipam.org.br, erikappp@yahoo.com.br
} 
A degradação ambiental e a intrusão de novos elementos culturais acompanhados pela desagregação dos sistemas de vida tradicionais ameaçam, além de um acervo de conhecimentos empíricos, um patrimônio genético de valor inestimável para as futuras gerações (Amorozo \& Gely 1988). Outra ameaça deve-se ao fato da pesquisa científica sobre plantas utilizadas por comunidades tradicionais brasileiras ser recente, sendo assim, pouco documentada, aliada à forma delicada como este conhecimento é mantido, através da tradição oral.

Portanto, este trabalho teve como objetivos: a) realizar estudo etnobotânico das espécies utilizadas para fins medicinais pelas comunidades rurais da Marambaia e Camboinha (Itacaré, BA); b)avaliar a relevância deste conhecimento para as comunidades em questão; c) retornar o trabalho finalizado aos representantes das comunidades estudadas e aos responsáveis pelo projeto desenvolvido pelo IESB (Instituto de Estudos Sócio-Ambientais do Sul da Bahia) para o desenvolvimento sustentável da região de Itacaré - Serra Grande, com o fim de contribuir na valorização do conhecimento local; d) fornecer subsídios para estudos etnofarmacológicos que promovam a interação de sistemas médicos populares e modernos, propiciando melhor aproveitamento e maior segurança no uso dos recursos terapêuticos locais.

\section{Material e métodos}

A área de estudo localiza-se ao Norte da costa do Cacau (Sul da Bahia) e pertence à Área de Proteção Ambiental (APA) Itacaré/Serra Grande (Fig. 1). A APA delimita-se ao Norte pela foz do Rio de Contas (Município de Itacaré); ao sul, pelo riacho Sergi (limite entre os Municípios de Uruçuca e Ilhéus); a oeste, numa linha eqüidistante a $6 \mathrm{~km}$ da linha de preamar e, a leste, pelo Oceano Atlântico - Art $1^{\circ}$ do decreto $\mathrm{n}^{\mathrm{o}} 2.186$ de 07/06/1993 (Plano de manejo da APA Itacaré/Serra Grande 1998). Em 2004, dois anos após o término do presente estudo, houve a ampliação dos limites da APA, como pode ser observado na Fig. 1.

Anteriormente à transformação das áreas em assentamentos rurais na década de 1980 (informalmente no caso da Camboinha ou através da Reforma Agrária, no caso da Marambaia), toda a paisagem local era formada pela Floresta Ombrófila Densa. A introdução das "roças" (termo empregado no sentido de sítios) de subsistência e pastagens levou ao desmatamento gradual de áreas de floresta devido à baixa capacidade produtiva do solo e à utilização de técnicas agrícolas inadequadas, que geravam uma necessidade constante de derrubar novas áreas para o aproveitamento de solos mais férteis para novos plantios. Outro fator que causou considerável dano à biodiversidade e aos recursos naturais da região foi a pavimentação da Rodovia Ilhéus-Itacaré, em 1998, que tornou a região exposta a problemas ambientais oriundos do desmatamento para construção de empreendimentos turísticos, da exploração clandestina de madeira nativa, da deposição inadequada de lixo em áreas de mata, trilhas, cachoeiras e praias, entre outros (Silva Filho 2000).

Os habitantes das comunidades estudadas se caracterizam de um modo geral, pela origem essencialmente rural. Em sua maioria, nasceram no sul da Bahia ou em municípios do semi-árido baiano (Silva Filho 2000). Atualmente, as principais atividades econômicas são: agricultura, comercialização dos produtos da roça, atividades ligadas ao "ecoturismo" local e à conservação da mata. Nas comunidades não existem postos de atendimento à saúde. Quando os moradores precisam de algum atendimento médico, têm que se deslocar até a cidade de Itacaré ou outras cidades próximas, muitas vezes caminhando (o que leva no mínimo uma hora e meia, ou mais, dependendo do local onde moram).

No trabalho de campo, realizado nos meses de janeiro, fevereiro e julho/2002, foram feitas visitas a 26 sítios (correspondendo a cerca de $24 \%$ de um total de 107 sítios: 75 na Camboinha e 32 na Marambaia), 11 sítios localizados na comunidade da Marambaia e 15 na comunidade da Camboinha. Devido à inexistência de um mapa de distribuição dos sítios na área e à dificuldade de acesso a alguns deles, não foi

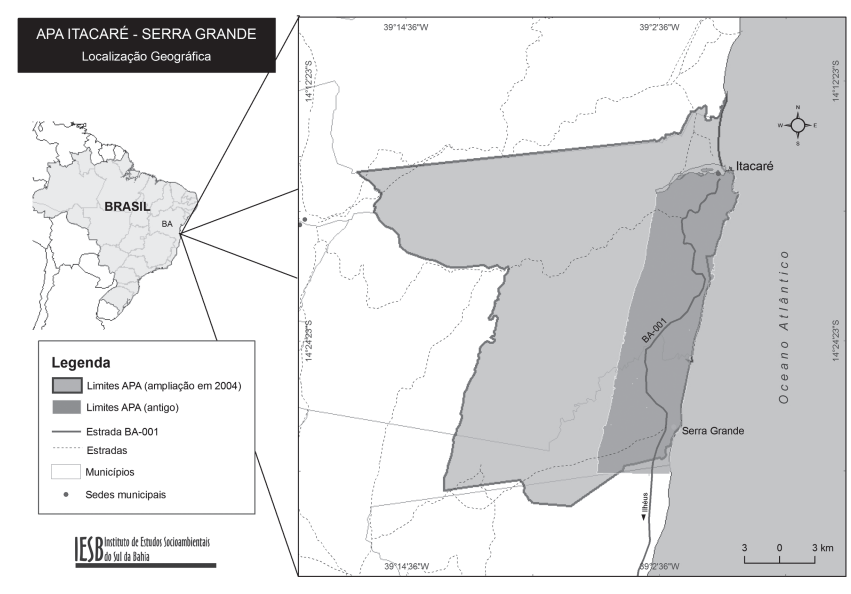

Figura 1. Mapa da área de estudo. Fonte: Base de Dados IESB (Instituto de Estudos Socioambientais do Sul da Bahia). 
possível fazer uma amostra aleatória. Foram escolhidos oito ramais ou vias de acesso, ao longo dos quais localizam-se os sítios visitados. O tamanho da amostra foi definido em campo com o auxílio da "curva do coletor" (Krebs 1989).

Nas primeiras visitas, foram apresentados às famílias o projeto e seus objetivos. A coleta de informações deu-se por meio de entrevistas não estruturadas, semi-estruturadas e estruturadas (Bernard 1988; Martin 1995), para levantamento de dados: a) sócio-econômicos dos informantes; b) etnobotânicos (nome popular, partes usadas, formas de preparo, dosagens, aplicação, fins terapêuticos, restrições); c) grau de importância (preferência) e/ou necessidade do uso da medicina popular em relação à medicina convencional; d) participação do informante nos projetos monitorados pelo IESB e sua percepção em relação à conservação da biodiversidade local e às mudanças advindas com a implantação da APA Itacaré/ Serra Grande. Adotou-se como critério, para a coleta de dados, entrevistar a pessoa mais velha de cada domicílio.

As amostras vegetais coletadas foram preparadas segundo Ming (1996), colocadas em estufa para secagem, identificadas por taxonomista (A. Furlan) por meio de comparação com exsicatas do herbário HRCB e depositadas no mesmo. Os dados indicados pelos entrevistados foram registrados em caderneta de campo.

As doenças tratadas com as plantas medicinais foram classificadas de acordo com o CID 10 (Classificação Estatística Internacional de Doenças e Problemas Relacionados à Saúde, OMS 2000) .

Calcularam-se os índices de concordância de uso (Amorozo \& Gely 1988), de diversidade (Begossi 1996; Krebs 1989) e de eqüitabilidade (Magurran 1989). A porcentagem de concordância quanto aos usos principais para cada espécie (CUP) mostra a importância relativa das plantas utilizadas nestas comunidades quanto ao número de informantes que as citaram e à concordância dos usos citados (aqui foram consideradas as espécies citadas por cinco ou mais informantes). Para isso, foram realizados os seguintes cálculos para cada espécie (Amorozo \& Gély 1988):

$$
\mathrm{CUP}=(\mathrm{ICUP} / \mathrm{ICUE}) \times 100
$$

onde: ICUP $=$ número de informantes citando o uso principal da espécie: ICUE = número total de informantes citando uso da espécie.

Calculou-se o fator de correção (FC) para cada espécie, o qual permite a extração de valores de importância relativos à espécie mais citada pelos informantes (CUPc).
$\mathrm{FC}=\mathrm{ICUE} / \mathrm{ICEMC} \quad \mathrm{CUPc}=\mathrm{CUP} \times \mathrm{FC}$ onde: $\mathrm{ICEMC}=$ número de informantes que citaram a espécie mais citada.

O índice de diversidade de Shannon-Wiener e o índice de eqüitabilidade permitem comparações entre a diversidade do conhecimento etnobotânico de diferentes comunidades e, em geral, auxiliam no entendimento de suas interações com o ambiente (Begossi 1996). A fórmula usada para calcular o índice de diversidade de Shannon-Wiener é a seguinte (Krebs 1989): $\mathrm{S}$

$$
\mathrm{H}^{\prime}=-\underset{\mathrm{i}=1}{\sum(\mathrm{pi})\left(\log ^{2} \mathrm{pi}\right)}
$$

onde: $\mathrm{S}=$ número de espécies; $\mathrm{pi}=\mathrm{ni} / \mathrm{N} ; \mathrm{ni}=$ número de citações por espécie; $\mathrm{N}$ = número total de citações; $\mathrm{H}^{\prime}=$ índice de diversidade.

O índice de eqüitabilidade ou uniformidade é dado por $\mathrm{H}^{\prime} / \log ^{2} \mathrm{~S}$ (Krebs 1989). Ambos os índices foram calculados usando-se o programa Krebs para Windows (1997). Para verificar suficiência amostral, usou-se o método de rarefação, calculado através do Programa computacional Krebs Ecological Methodology - versão para o MS-DOS (Krebs 1989). Os dados obtidos a partir das entrevistas abertas foram submetidos a análises qualitativas.

\section{Resultados e discussão}

Todos os entrevistados (21 mulheres e 5 homens) nasceram no Estado da Bahia, sendo que metade mora há mais de 15 anos na área. Dos 26 entrevistados, $81 \%$ são de origem rural e $33 \%$ afirmaram ser descendentes de índios. A maioria (70\%) situa-se na faixa etária entre 35 e 64 anos. A renda familiar mensal chega no máximo a 200 reais ( $73 \%$ dos entrevistados), e em $27 \%$ dos domicílios moram mais que seis pessoas. Quanto à escolaridade dos entrevistados, $42 \%$ nunca estudaram e $54 \%$ nem chegaram a completar o primeiro grau. Ainda, $61 \%$ são evangélicos.

A agricultura é a principal atividade de 53,8\% dos entrevistados. Outros tipos de ocupação citados foram: serviços do lar (apenas no caso das mulheres), comércio de produtos da roça, guia de trilhas (alternativa econômica recente na região), vendedores, entre outros. Mesmo quando não é a principal fonte de renda, a agricultura figura entre as atividades das famílias. Em geral, os moradores cultivam em suas roças: coco, aipim, banana, jaca, hortaliças, graviola, abacaxi, mamão, manga, cana, laranja, abacate, caju, limão, cacau, cajarana, entre outras. 
Aproximadamente $74 \%$ das 98 espécies citadas pelos informantes são cultivadas, sendo mais da metade exóticas. Vale ressaltar a pequena representatividade da flora de mata atlântica (apenas cinco ou seis espécies). Este resultado é intrigante, tendo-se em vista a riqueza de espécies tropicais nativas na área e a ascendência indígena de cerca de um terço da população amostrada. Situação semelhante é registrada por Voeks (1996) em outra área de Mata Atlântica na Bahia: uma flora medicinal fundamentalmente herbácea, cultivada e exótica. Espécies comumente cultivadas em jardins (Gossypium barbadense L., Ruta graveolens L., Mentha spp., por exemplo) são comuns a ambos os trabalhos. As razões que podem explicar tal fato são complexas: prendem-se, de um lado, à introdução e popularização de espécies da Europa, África e Ásia, desde tempos coloniais, além das mudanças culturais e da miscigenação étnica, ao longo de cinco séculos de colonização (Voeks 1996; Bennett $\&$ Prance 2000). Por outro lado, a facilidade de cultivar plantas medicinais ao redor das casas, aliada ao fato de que o uso de plantas medicinais está predominante entre as mulheres destas comunidades, que não têm o costume de ir mata adentro coletar plantas nativas, especialmente por medo de serpentes, também pode ajudar a fixar este padrão de exploração preferencial das plantas exóticas cultivadas.

As espécies herbáceas, cultivadas ou espontâneas, representam $51,1 \%$ de toda a porção amostral $(\mathrm{n}=98)$. Em seguida, têm-se as espécies de hábito arbóreo $(21,4 \%)$, subarbustivo $(21,4 \%)$ e arbustivo $(6,1 \%)$.

Em geral, as plantas medicinais citadas não necessitam de muitos cuidados e quando adubadas, isso é feito de forma orgânica (restos de frutos e folhas). As cultivadas se encontram, quase sempre, próximas à casa (em vasos de casca de coco, latas ou outros materias reutilizáveis, em canteiros ou no próprio solo). As espontâneas costumam crescer em beiras de estrada e áreas desprovidas de vegetação, com insolação direta na maior parte do dia.

A Tabela 1 apresenta 105 diferentes nomes populares atribuídos a 98 espécies de uso medicinal citadas pelas comunidades, suas formas de manejo (cultivadas ou espontâneas), o número de informantes que citou cada uma das espécies e o número de usos atribuídos para cada espécie.

As espécies pertencem a 40 famílias, sendo que só Lamiaceae, Verbenaceae, Asteraceae e Rutaceae, representam juntas $30,6 \%$ das espécies. A família Lamiaceae é representada por 12 espécies e cada uma das outras, acima citadas, por seis (Fig. 2). Estas são famílias com muitas espécies que contêm substâncias com atividade biológica. Há evidências de que a seleção de plantas para uso medicinal não é feita ao acaso e que famílias botânicas com compostos bioativos tendem a ser mais bem representadas nas farmacopéias populares (Moerman \& Estabrook 2003). Dentre as sete famílias com maior representatividade em espécies introduzidas nas farmacopéias de vários grupos nativos do norte da América do Sul (Bennett \& Prance 2000), seis encontram-se também entre as mais representativas nas comunidades da Marambaia e Camboinha: Lamiaceae, Asteraceae, Rutaceae, Fabaceae, Poaceae e Apiaceae. Segundo Bennett \& Prance (2000), estas são famílias que podem ser encontradas tanto em clima tropical como temperado, e dominam o grupo de plantas medicinais introduzidas. Lamiaceae e Asteraceae destacam-se ainda em diferentes regiões do Brasil, contribuindo com o maior número de espécies em outras áreas de mata atlântica (Hanazaki et al. 2000), no semi-árido pernambucano (Almeida \& Albuquerque 2002) e na Estação Ecológica de Jataí, SP (Castellucci et al. 2000), onde são, às vezes, também as mais representativas quanto ao número de usos (Marodin \& Baptista 2002).

As plantas mais citadas foram o mastruz (Chenopodium ambrosioides L.) e a erva-cidreira (Lippia alba (Mill) N.E. Br.). O número de usos terapêuticos relacionado a estas espécies está também entre os maiores. A folha é a parte do vegetal significantemente mais utilizada na medicina caseira local ( $73 \%$ dos casos), para o tratamento de todas as doenças citadas, seguida por raízes $(6,8 \%)$, flores $(3,5 \%)$, planta inteira $(2,9 \%)$, frutos $(2,8 \%)$, bulbo $(1,9 \%)$, sementes $(1,6 \%)$, cascas $(1,5 \%)$, folhas sem nervura central ( $1 \%)$ e outros. A forma de preparação mais utilizada é o chá, por decocção $(59,1 \%$ dos casos). As preparações medicinais são feitas também na forma de banhos $(9,9 \%)$, macerados $(8,7 \%)$, xaropes $(6,9 \%)$, compressas $(3,3 \%)$, sucos $(3,1 \%)$, cataplasmas $(2,3 \%)$, tinturas $(2,1 \%)$, in natura $(1,8 \%)$, e outros.

As doenças foram categorizadas de acordo com o CID-10 - Classificação estatística internacional de doenças e problemas relacionados à saúde (Organização Mundial da Saúde 2000). A Tab. 2 apresenta a porcentagem de citações $(n=519)$ de doenças em cada categoria. Ainda, dentro de cada categoria, a representatividade da doença mais citada. O termo "doenças culturais" refere-se às manifestações interpretadas como doenças e que não apresentam uma causa fundamentada cientificamente. 
Tabela 1. Espécies usadas para fins medicinais $(\mathrm{n}=98)$ pelas comunidades da Marambaia e Camboinha (Itacaré, BA). Ref $=$ número da coleta ou do herbário; $(\mathrm{EP}=$ Erika Pinto $) ; \mathrm{C}=$ cultivada; $\mathrm{E}=$ espontânea; ICUE = informantes citando uso da espécie; NUC = número de usos citados para cada espécie; indet = indeterminado.

\begin{tabular}{|c|c|c|c|c|c|}
\hline Nome científico & Nome popular & Ref & $\mathrm{C} / \mathrm{E}$ & ICUE & NUC \\
\hline \multicolumn{6}{|l|}{ ACANTHACEAE } \\
\hline cf. Justicia sp. 1 & abre-caminho & EP71 & $\mathrm{C}$ & 1 & 1 \\
\hline cf. Justicia sp. 2 & emburana & EP64 & $\mathrm{C}$ & 1 & 1 \\
\hline \multicolumn{6}{|l|}{ AMARANTHACEAE } \\
\hline Alternanthera brasiliana (L.) Kuntze & ampecilina, anador I, bezetacil, dipurana, novalgina I & 38619 & $\mathrm{C}$ & 4 & 5 \\
\hline Pfaffia glomerata Spreng. & novalgina II, doril & 38615 & $\mathrm{C} / \mathrm{E}$ & 4 & 4 \\
\hline \multicolumn{6}{|l|}{ ANACARDIACEAE } \\
\hline Anacardium occidentale $\mathrm{L}$. & caju & EP41 & $\mathrm{C}$ & 8 & 3 \\
\hline Mangifera indica $\mathrm{L}$. & manga & EP02 & $\mathrm{C}$ & 1 & 1 \\
\hline \multicolumn{6}{|l|}{ ANNONACEAE } \\
\hline Rollinia sericea R.E. Fries & graviola & EP01 & $\mathrm{C}$ & 7 & 4 \\
\hline \multicolumn{6}{|l|}{ APIACEAE } \\
\hline Daucus carota $\mathrm{L}$. & cenoura & EP97 & $\mathrm{C}$ & 2 & 2 \\
\hline Foeniculum vulgare Miller. & erva-doce & 38610 & $\mathrm{C}$ & 1 & 1 \\
\hline Petroselinum crispum (Mill.) & salsa & EP92 & $\mathrm{C}$ & 1 & 1 \\
\hline \multicolumn{6}{|l|}{ ARECACEAE } \\
\hline Cocos nucifera $\mathrm{L}$. & coco & EP84 & $\mathrm{C}$ & 3 & 3 \\
\hline \multicolumn{6}{|l|}{ ASTERACEAE } \\
\hline Artemisia sp. & cravo-de-defunto & EP86 & $\mathrm{C}$ & 1 & 2 \\
\hline Bidens pilosa $\mathrm{L}$. & picão, carrapicho-agulha & 38614 & $\mathrm{E}$ & 12 & 8 \\
\hline Lactuca sativa $\mathrm{L}$. & alface & EP89 & $\mathrm{C}$ & 1 & 1 \\
\hline Sonchus oleraceus L. & sarraia & EP79 & $\mathrm{E}$ & 1 & 1 \\
\hline Vernonia condensata Baker & alumã & 38621 & $\mathrm{C}$ & 7 & 6 \\
\hline Wedelia paludosa DC. & mal-me-quer & 38633 & $\mathrm{E}$ & 6 & 9 \\
\hline \multicolumn{6}{|l|}{ BORAGINACEAE } \\
\hline cf. Cordia sp. & maria-preta & 38632 & $\mathrm{E}$ & 6 & 6 \\
\hline Symphytum officinale L. & confrei & EP66 & $\mathrm{C}$ & 2 & 3 \\
\hline \multicolumn{6}{|l|}{ BRASSICACEAE } \\
\hline Brassica oleracea L. & couve-branco & EP91 & $\mathrm{C}$ & 1 & 2 \\
\hline \multicolumn{6}{|l|}{ CAESALPINACEAE } \\
\hline Senna occidentalis (L.) Link & fedegoso & 38616 & $\mathrm{C}$ & 6 & 6 \\
\hline \multicolumn{6}{|l|}{ CAPPARACEAE } \\
\hline Cleome affinis DC. & xixi-de-galinha & 38611 & $\mathrm{C}$ & 6 & 3 \\
\hline \multicolumn{6}{|l|}{ CAPRIFOLIACEAE } \\
\hline Sambucus nigra L. & sabugueiro & EP73 & $\mathrm{C}$ & 2 & 4 \\
\hline \multicolumn{6}{|l|}{ CECROPIACEAE } \\
\hline Cecropia pachystachia Trec. & embaúba & EP68 & $\mathrm{E}$ & 2 & 2 \\
\hline \multicolumn{6}{|l|}{ CHENOPODIACEAE } \\
\hline Beta vulgaris $\mathrm{L}$. & beterraba & EP96 & $\mathrm{C}$ & 2 & 3 \\
\hline Chenopodium ambrosioides $\mathrm{L}$. & mastruz & EP13 & $\mathrm{E}$ & 22 & 14 \\
\hline \multicolumn{6}{|l|}{ CRASSULACEAE } \\
\hline Kalanchoe pinnata Pers. & folha-da-costa & 38618 & $\mathrm{C}$ & 6 & 6 \\
\hline \multicolumn{6}{|l|}{ CYPERACEAE } \\
\hline Rhynchospora nervosa Boeck & capim-estrela & 38622 & $\mathrm{E}$ & 4 & 5 \\
\hline \multicolumn{6}{|l|}{ EUPHORBIACEAE } \\
\hline Euphorbia cf. tirucalli L. & graveto & EP70 & $\mathrm{C}$ & 1 & 1 \\
\hline Manihot esculenta Crantz. & mandioca & EP55 & $\mathrm{C}$ & 1 & 2 \\
\hline cf. Sebastiana sp. & catinga-de-bode & 38630 & $\mathrm{C}$ & 1 & 1 \\
\hline \multicolumn{6}{|l|}{ FABACEAE } \\
\hline Aeschynomene sp. & desinchadeira & 38631 & $\mathrm{E}$ & 1 & 1 \\
\hline Crotalaria micans Link. & andu & 38617 & $\mathrm{E}$ & 1 & 1 \\
\hline cf. Crotalaria sp. & desinchadeira pandeirinho & 38627 & $\mathrm{C}$ & 1 & 1 \\
\hline Desmodium cf. adscendens (SW) DC. & carrapicho-chato & EP47 & $\mathrm{E}$ & 1 & 1 \\
\hline Zornia latifolia $\mathrm{Sm}$. & arrozinho & 38625 & $\mathrm{E}$ & 5 & 3 \\
\hline
\end{tabular}


Tabela 1 (continuação)

\begin{tabular}{|c|c|c|c|c|c|}
\hline Nome científico & Nome popular & Ref & $\mathrm{C} / \mathrm{E}$ & ICUE & NUC \\
\hline \multicolumn{6}{|l|}{ LAMIACEAE } \\
\hline Leonotis nepetaefolia (L.) R. Br. & cordão-de-são-francisco & 38608 & $\mathrm{C}$ & 2 & 2 \\
\hline Mentha pulegium $\mathrm{L}$. & poejo & EP30 & $\mathrm{C}$ & 1 & 1 \\
\hline Mentha sp. 1 & hortelã & EP31 & $\mathrm{C}$ & 6 & 5 \\
\hline Mentha sp. 2 & hortelã-miúdo & EP06 & $\mathrm{C}$ & 2 & 2 \\
\hline cf. Mentha sp. & água-de-alevante & EP27 & $\mathrm{C}$ & 5 & 6 \\
\hline Ocimum basilicum $\mathrm{L}$. & manjericão & 38628 & $\mathrm{C}$ & 5 & 5 \\
\hline Ocimum sp. & alfavaca-de-galinha, alfavaca-fina & EP12 & $\mathrm{C}$ & 7 & 3 \\
\hline Ocimum cf. gratissimum L. & quioiô & 38606 & $\mathrm{C}$ & 7 & 5 \\
\hline Ocimum selloi Benth. & alixis, alixis-paragó, anador III & 38605 & $\mathrm{C}$ & 4 & 4 \\
\hline Plectranthus cf. amboinicus Lour. & alfavaca-grossa, hortelã-grosso & EP07 & $\mathrm{C}$ & 10 & 6 \\
\hline Plectranthus cf. barbatus Andrews. & folha-de-santa-bárbara, oxalá & EP10 & $\mathrm{C}$ & 8 & 6 \\
\hline Plectranthus neochilus Schlechter. & boldo & EP05 & $\mathrm{C}$ & 13 & 10 \\
\hline \multicolumn{6}{|l|}{ LAURACEAE } \\
\hline Persea americana Mill. & abacate & EP52 & $\mathrm{C}$ & 9 & 7 \\
\hline \multicolumn{6}{|l|}{ LILIACEAE } \\
\hline Allium cepa $\mathrm{L}$. & cebola & EP93 & $\mathrm{C}$ & 3 & 3 \\
\hline Allium sativum $\mathrm{L}$. & alho & EP90 & $\mathrm{C}$ & 5 & 8 \\
\hline Aloe vera (L.) Burm. F. & babosa & EP98 & $\mathrm{C}$ & 2 & 2 \\
\hline \multicolumn{6}{|l|}{ MALVACEAE } \\
\hline Gossypium barbadense L. & algodão & 38624 & $\mathrm{C}$ & 11 & 13 \\
\hline \multicolumn{6}{|l|}{ MELASTOMATACEAE } \\
\hline Miconia albicans Stewd. & canela-de-velho & EP82 & $\mathrm{E}$ & 1 & 2 \\
\hline \multicolumn{6}{|l|}{ MIRISTICACEAE } \\
\hline Myristica fragans Houtt. & noz-moscada & EP74 & $\mathrm{C}$ & 2 & 4 \\
\hline \multicolumn{6}{|l|}{ MUSACEAE } \\
\hline Musa acuminata Collad. & banana & EP80 & $\mathrm{C}$ & 3 & 7 \\
\hline \multicolumn{6}{|l|}{ MYRTACEAE } \\
\hline Eugenia uniflora L. & pitanga & EP33 & $\mathrm{C}$ & 5 & 3 \\
\hline Eugenia sp. & jambo & EP25 & $\mathrm{C}$ & 1 & 1 \\
\hline Psidium guajava $\mathrm{L}$. & goiaba & EP95 & $\mathrm{C}$ & 1 & 1 \\
\hline Psidium sp. & araçá & EP24 & $\mathrm{E}$ & 4 & 2 \\
\hline \multicolumn{6}{|l|}{ OXALIDACEAE } \\
\hline Averrhoa bilimbi L. & Biri-biri & EP83 & $\mathrm{C}$ & 1 & 2 \\
\hline \multicolumn{6}{|l|}{ PIPERACEAE } \\
\hline Peperomia pellucida Kunth & alfavaquinha-de-cobra & EP49 & $\mathrm{E}$ & 4 & 4 \\
\hline Pothomorphe peltata (L.) Miq. & capeba & EP34 & $\mathrm{E}$ & 2 & 2 \\
\hline \multicolumn{6}{|l|}{ PLANTAGINACEAE } \\
\hline Plantago major L. & tanchagem & EP43 & $\mathrm{E}$ & 3 & 4 \\
\hline \multicolumn{6}{|l|}{ POACEAE } \\
\hline Cymbopogon citratus Stapf. & capim-santo & EP37 & $\mathrm{C}$ & 12 & 7 \\
\hline Digitaria insularis (L.) Fedde. & capim-açu & EP51 & $\mathrm{E}$ & 2 & 1 \\
\hline Melinis minutiflora Beauv. & capim-gordura & EP56 & $\mathrm{E}$ & 1 & 2 \\
\hline Sorghum cf. halepense L. Pers. & capim-aruanda & EP32 & $\mathrm{E}$ & 3 & 5 \\
\hline \multicolumn{6}{|l|}{ POLYGALACEAE } \\
\hline Polygala paniculata $\mathrm{L}$. & bangui, vassourinha, vicky & 38629 & $\mathrm{E}$ & 3 & 5 \\
\hline \multicolumn{6}{|l|}{ RUBIACEAE } \\
\hline Genipa americana $\mathrm{L}$. & jenipapo & EP50 & $\mathrm{C}$ & 4 & 4 \\
\hline Spermacoce verticillata $\mathrm{L}$. & carquejinha & 38620 & $\mathrm{E}$ & 2 & 2 \\
\hline \multicolumn{6}{|l|}{ RUTACEAE } \\
\hline Citrus aurantiifolia Swingle & limão-merim & EP54 & $\mathrm{C}$ & 2 & 4 \\
\hline Citrus limon (L.) Burm. F. & limão-rosa & EP53 & $\mathrm{C}$ & 1 & 1 \\
\hline Citrus reticulata Blanco & tangerina & EP69 & $\mathrm{C}$ & 1 & 1 \\
\hline Citrus sinensis (L.) Osbeck. & laranja & EP03 & $\mathrm{C}$ & 9 & 5 \\
\hline Citrus sp. & limão-balão & EP58 & $\mathrm{C}$ & 1 & 1 \\
\hline Ruta graveolens L. & arruda & EP26 & $\mathrm{C}$ & 8 & 7 \\
\hline \multicolumn{6}{|l|}{ SCROPHULARIACEAE } \\
\hline Scoparia dulcis L. & vassourinha II & 38612 & $\mathrm{E}$ & 3 & 2 \\
\hline
\end{tabular}


Tabela 1 (continuação)

\begin{tabular}{|c|c|c|c|c|c|}
\hline Nome científico & Nome popular & Ref & $\mathrm{C} / \mathrm{E}$ & ICUE & NUC \\
\hline \multicolumn{6}{|l|}{ SOLANACEAE } \\
\hline Solanum americanum Mill. & erva-de-santa-maria & EP88 & $\mathrm{E}$ & 1 & 1 \\
\hline Solanum paniculatum L. & jurubeba & EP72 & $\mathrm{E}$ & 2 & 4 \\
\hline Solanum tuberosum L. & batatinha & EP77 & $\mathrm{C}$ & 3 & 2 \\
\hline \multicolumn{6}{|l|}{ URTICACEAE } \\
\hline Pilea microphylla (L.) Liebm & brilhantina & EP75 & $\mathrm{C}$ & 1 & 1 \\
\hline \multicolumn{6}{|l|}{ VERBENACEAE } \\
\hline indet. & alfazema II & 38626 & $\mathrm{C}$ & 1 & 4 \\
\hline indet. & narapiró & EP61 & $\mathrm{C}$ & 1 & 1 \\
\hline Lantana camara $\mathrm{L}$. & camará & 38623 & $\mathrm{E}$ & 3 & 2 \\
\hline Lippia alba (Mill) N.E. Br. & erva-cidreira & 38609 & $\mathrm{C}$ & 17 & 13 \\
\hline Lippia $\mathrm{sp}$ & melissa & EP78 & $\mathrm{C}$ & 2 & 4 \\
\hline Stachytarpheta cayennensis (Rich) Vahl. & chá-de-burro & 38607 & $\mathrm{E}$ & 1 & 1 \\
\hline \multicolumn{6}{|l|}{ VIOLACEAE } \\
\hline cf. Hybanthus sp. & pulga-do-campo & EP35 & $\mathrm{E}$ & 9 & 8 \\
\hline \multicolumn{6}{|l|}{ ZINGIBERACEAE } \\
\hline Alpinia sp. & água-de-colônia & EP87 & $\mathrm{C}$ & 2 & 2 \\
\hline Costus spiralis & cana-de-macaco & EP59 & $\mathrm{C}$ & 5 & 3 \\
\hline cf. Hedychium sp. 1 & jasmim & EP76 & $\mathrm{C}$ & 2 & 3 \\
\hline cf. Hedychium sp. 2 & lepurdina & EP40 & $\mathrm{C}$ & 3 & 3 \\
\hline \multicolumn{6}{|l|}{ A confirmar $(\mathrm{cf})$} \\
\hline cf. Acanthaceae & anador II & EP63 & $\mathrm{C}$ & 1 & 1 \\
\hline cf. Amaranthaceae & caminho-de-roça & EP45 & $\mathrm{C}$ & 1 & 1 \\
\hline cf. Apiaceae & alfazema I & EP08 & $\mathrm{C}$ & 1 & 2 \\
\hline cf. Lamiaceae & mãe-boa & EP17 & $\mathrm{C}$ & 1 & 1 \\
\hline
\end{tabular}

As doenças mais citadas que podem ser tratadas a partir das plantas medicinais utilizadas pelas comunidades estão na categoria das "Doenças, sintomas e sinais relativos ao aparelho digestivo e abdome" (21,4\%). Dentro dessa categoria, o sintoma mais citado foi o de dor de barriga. A ausência de tratamento de água usada para os diversos fins nas comunidades pode explicar estes resultados. Em seguida, têm-se sintomas e sinais gerais $(15,4 \%)$ e doenças do aparelho respiratório $(10,8 \%)$.

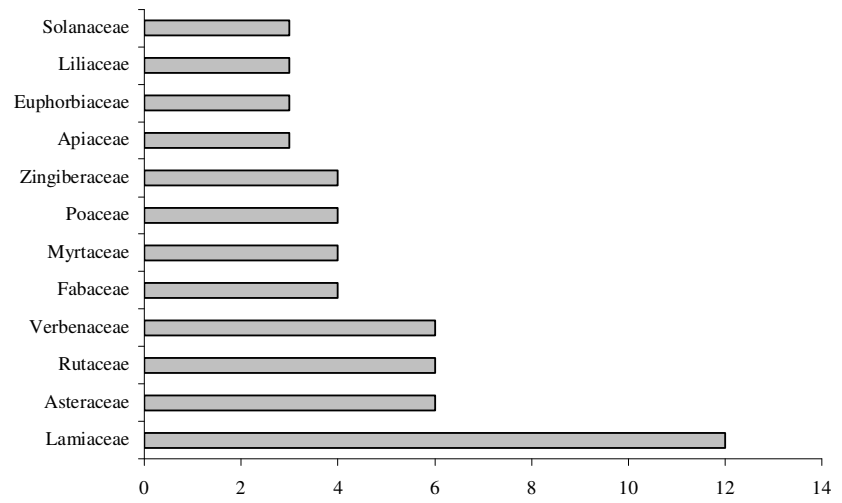

Figura 2. Famílias mais representativas das espécies usadas pelas comunidades da Marambaia e Camboinha.
Di Stasi (2002), em trabalho também realizado em região de Mata Atlântica, encontrou resultados semelhantes. Doenças relacionadas ao sistema gastrointestinal e ao respiratório estão entre as mais citadas, com $18,3 \%$ e $18,5 \%$ do total de citações, respectivamente. Trabalhos realizados no Estado de Pernambuco (Almeida \& Albuquerque 2002) e Mato Grosso (Amorozo 2002) registraram também um maior número de citações para transtornos do aparelho digestivo, respiratório e geniturinário. No presente trabalho, gripe e tosse estão entre as doenças e/ou sintomas mais citados dentro de suas categorias com percentual elevado de 64,3 e 75,1 respectivamente.

Uma planta com um índice de concordância relativamente alto, isto é, que tenha vários informantes concordando com um mesmo uso terapêutico, talvez possa sugerir uma real efetividade no tratamento da doença (Friedman et al. 1986). Em um estudo etnobotânico, o índice facilitará a seleção de espécies para testes farmacológicos que possam vir a comprovar uma real eficácia de seus princípios ativos.

Pode-se observar um índice de concordância de uso (CUP) alto (de $80 \%$ ou mais) para a graviola (Rollinia sericea R.E. Fries.), o quioiô (Ocimum cf. gratissimum L.) e a pitanga (Eugenia uniflora L.) 
Tabela 2. Porcentagem de citações para cada categoria ( $\mathrm{n}=519)$. Doença mais citada em cada categoria e sua porcentagem dentro desta.

\begin{tabular}{|c|c|c|c|}
\hline Categorias & \% de citações & $\begin{array}{l}\text { Doença mais citada } \\
\text { dentro da categoria }\end{array}$ & $\begin{array}{c}\% \text { de citações } \\
\text { da doença mais citada }\end{array}$ \\
\hline $\begin{array}{l}\text { Doenças, sintomas e sinais relativos ao aparelho digestivo e } \\
\text { abdome }\end{array}$ & 21,4 & dor de barriga & 18,0 \\
\hline Sintomas e sinais gerais & 15,4 & febre & 41,3 \\
\hline Doenças do aparelho respiratório & 10,8 & gripe & 64,3 \\
\hline $\begin{array}{l}\text { Lesões, envenenamento e algumas outras conseqüências de } \\
\text { causas externas }\end{array}$ & 8,3 & pancada & 30,2 \\
\hline Doenças do aparelho geniturinário & 7,7 & rim & 30,0 \\
\hline Outros & 7,3 & inflamação & 81,6 \\
\hline Sintomas e sinais relativos ao aparelho circulatório e respiratório & 5,4 & tosse & 75,1 \\
\hline Doenças do aparelho circulatório & 3,8 & pressão alta & 44,9 \\
\hline "Doenças culturais" & 3,5 & mau-olhado & 61,1 \\
\hline Doenças infecciosas intestinais & 3,3 & verme & 82,6 \\
\hline Outras doenças infecciosas e parasitárias & 3,1 & hepatite & 56,2 \\
\hline Doenças da pele e do tecido subcutâneo & 3,1 & queda de cabelo & 37,7 \\
\hline Doenças endócrinas, nutricionais e metabólicas & 2,1 & diabete & 45,3 \\
\hline Doenças do ouvido & 1,3 & dor de ouvido & 100 \\
\hline Doenças do olho & 1,3 & olhos doentes & 85,9 \\
\hline Gravidez, parto e puerpério & 0,8 & puerpério & 75,3 \\
\hline Neoplasias & 0,6 & câncer & 65,5 \\
\hline Doenças do sangue e dos órgãos hematopoéticos & 0,6 & anemia & 100 \\
\hline Doenças do sistema osteomuscular e do tecido conjuntivo & 0,2 & reumatismo & 100 \\
\hline
\end{tabular}

(Tab. 3). Porém, o número de informantes que citaram seu uso foi relativamente baixo (entre cinco e sete). Das 30 espécies citadas pelo menos por cinco informantes, apenas $30 \%$ apresentam um CUP inferior a 50\%. O mastruz (Chenopodium ambrosioides L.), a mais citada, tem como uso principal o tratamento para vermes, mas apenas $45,4 \%$ dos informantes citaram este fim terapêutico para a espécie. Em outros locais, como em Barcarena, PA (Amorozo \& Gely 1988) e na comunidade pesqueira da Ilha dos Búzios, no litoral paulista (Begossi et al. 1993), o mastruz (Chenopodium ambrosioides L.) é também usado principalmente contra vermes. Neste último local, é também a espécie mais utilizada medicinalmente. A erva-cidreira (Lippia alba (Mill) N.E. Br.), a segunda espécie mais citada, apresenta também um CUP alto. De todos os informantes, $76,5 \%$ citaram seu uso para dor de barriga (má digestão).

O valor do CUPc é, em geral, mais baixo que o CUP, pois é relativo à planta com maior número de informantes citando-a - no caso, o mastruz (Chenopodium ambrosioides L.). O CUPc para a graviola (Rollinea sericea R.E. Fries), espécie que apresenta o valor mais alto de CUP $(85,7 \%)$ para picada de cobra, cai para apenas $27,4 \%$.

A Tabela 4 mostra os índices de diversidade de Shannon-Wiener (base 10 e base e) e de equiitabilidade de diferentes áreas de estudos, os quais serão comparados e discutidos a seguir.

Os valores dos índices de diversidade de ShannonWiener para Itacaré (BA) estão bem próximos aos encontrados no Vale do Ribeira, SP (Di Stasi 2002). A equiitabilidade é relativamente alta, com valores próximos aos dos trabalhos citados na Tab. 4.

Dos locais acima citados, Barcarena, PA (Amorozo 2002) - área de floresta amazônica - é o que apresenta os maiores valores para os índices de diversidade e eqüitabilidade. Ainda, em Barcarena (Amorozo \& Gély 1988; Amorozo 1997), mais da metade das plantas coletadas crescem espontaneamente, muitas das quais são nativas do ambiente amazônico, ao contrário do presente trabalho, onde há apenas cerca de $26 \%$ de plantas espontâneas. As comunidades rurais de Itacaré e do Vale do Ribeira (Di Stasi 2002) possuem valores de diversidade bem próximos e ambas se encontram em área de Mata Atlântica. Sendo a área de estudo do presente trabalho provida de uma riqueza florística muito alta, equiparável à da floresta amazônica, apesar dos impactos ambientais aos quais a área está exposta, chama atenção o fato das comunidades presentes na área conhecerem um número muito elevado de plantas cultivadas para fins medicinais. Sendo ainda, 33\% dos informantes descendentes de índios, este quadro 
Tabela 3. Porcentagem de concordância quanto ao(s) uso(s) principal(is) (espécies citadas por cinco ou mais informantes). ICUE - $\mathrm{n}^{\circ}$ de informantes citando uso da espécie; ICUP - $\mathrm{n}^{\circ}$ de informantes citando uso principal; CUP - índice de concordância de uso; FC - fator de correção; CUPc - CUP corrigida.

\begin{tabular}{|c|c|c|c|c|c|c|}
\hline Nome científico & Uso principal & ICUE & ICUP & CUP & $\mathrm{FC}$ & CUPc \\
\hline Lippia alba (Mill) N.E. Br & dor de barriga, má digestão & 17 & 13 & 76,5 & 0,77 & 58,9 \\
\hline Chenopodium ambrosioides L. & vermes & 22 & 10 & 45,4 & 1,00 & 45,4 \\
\hline Plectranthus neochilus Schlechter & dor de barriga, má digestão & 13 & 10 & 76,9 & 0,59 & 45,4 \\
\hline Bidens pilosa $\mathrm{L}$. & hepatite & 12 & 07 & 58,3 & 0,54 & 31,5 \\
\hline Ocimum cf. gratissimum L. & gripe & 07 & 06 & 85,7 & 0,32 & 27,4 \\
\hline Rollinia sericea R.E. Fries & picada de cobra & 07 & 06 & 85,7 & 0,32 & 27,4 \\
\hline Cymbopogon citratus (DC.) Stapf. & gripe & 12 & 06 & 50,0 & 0,54 & 27,0 \\
\hline Plectranthus cf. amboinicus Lour. & gripe & 10 & 06 & 60,0 & 0,45 & 27,0 \\
\hline Ocimum sp. & gripe & 07 & 05 & 71,4 & 0,32 & 22,8 \\
\hline Anacardium occidentali $\mathrm{L}$. & cicatrizante & 08 & 05 & 62,5 & 0,36 & 22,5 \\
\hline Plecthranthus cf. barbatus Andrews & dor de barriga, má digestão & 08 & 05 & 62,5 & 0,36 & 22,5 \\
\hline Ruta graveolens $\mathrm{L}$. & olhos doentes & 08 & 05 & 62,5 & 0,36 & 22,5 \\
\hline Eugenia uniflora $\mathrm{L}$. & febre & 05 & 04 & 80,0 & 0,23 & 18,4 \\
\hline Vernonia condensata Baker. & dor de barriga, má digestão & 07 & 04 & 57,1 & 0,32 & 18,3 \\
\hline Citrus sinensis (L.) Osbeck & febre & 09 & 04 & 44,4 & 0,41 & 18,2 \\
\hline cf. Hybanthus sp. & inflamação & 09 & 04 & 44,4 & 0,41 & 18,2 \\
\hline Persea americana Mill & problema nos rins & 09 & 04 & 44,4 & 0,41 & 18,2 \\
\hline Wedelia paludosa DC. & machucado & 06 & 04 & 66,7 & 0,27 & 18,0 \\
\hline Allium sativum $\mathrm{L}$. & tosse & 05 & 03 & 60,0 & 0,23 & 13,8 \\
\hline Costus spiralis Rose. & hepatite & 05 & 03 & 60,0 & 0,23 & 13,8 \\
\hline Zornia latifolia $\mathrm{Sm}$. & inflamação & 05 & 03 & 60,0 & 0,23 & 13,8 \\
\hline Foeniculum vulgare Miller & calmante & 09 & 03 & 33,3 & 0,41 & 13,6 \\
\hline Gossypium barbadense L. & inflamação e machucado & 11 & 03 & 27,3 & 0,50 & 13,6 \\
\hline Cleome affinis DC. & inflamação & 06 & 03 & 50,0 & 0,27 & 13,5 \\
\hline Kalanchoe pinnata Pers. & tosse e frieira & 06 & 03 & 50,0 & 0,27 & 13,5 \\
\hline Mentha sp. 1 & vermes & 06 & 03 & 50,0 & 0,27 & 13,5 \\
\hline Senna occidentalis (L.) Link & febre & 06 & 03 & 50,0 & 0,27 & 13,5 \\
\hline cf. Mentha sp. 2 & inflamação e pós-parto & 05 & 02 & 40,0 & 0,23 & 9,2 \\
\hline Ocimum basilicum $\mathrm{L}$. & gripe e tosse & 05 & 02 & 40,0 & 0,23 & 9,2 \\
\hline cf. Cordia sp. & catarro e machucado & 06 & 02 & 33,3 & 0,27 & 9,0 \\
\hline
\end{tabular}

provavelmente deveria ser diferente.

A Figura 3 apresenta as curvas de rarefação para este trabalho e o de Barcarena, PA (Amorozo 2002). Embora os dois trabalhos tenham sido realizados em área de floresta tropical, em Barcarena (área de Floresta Amazônica) foi encontrado um número maior de espécies por tamanho amostral do que o encontrado em Itacaré (área de Floresta Atlântica). Talvez isso possa ser explicado pelo fato de que em Barcarena os esforços amostrais foram concentrados sobre os moradores que têm maior conhecimento sobre o uso de plantas medicinais (em Itacaré foi feito o levantamento do conhecimento geral das comunidades da Marambaia e Camboinha). Outro aspecto importante é que em Barcarena os moradores têm o costume de explorar diferentes ambientes, incluindo áreas de mata, para a coleta de espécies medicinais. Entretanto, em ambas as áreas observou-se que as

Tabela 4. Índices de diversidade em diferentes locais. NI = número de informantes; $\mathrm{NE}=$ número de espécies; $\mathrm{NC}=$ número de citações.

\begin{tabular}{|c|c|c|c|c|c|c|c|}
\hline \multirow[t]{2}{*}{ Local } & \multicolumn{2}{|c|}{ Índice de Shannon } & \multirow[t]{2}{*}{ Índice de eqüitabilidade } & \multirow[t]{2}{*}{ NI } & \multirow[t]{2}{*}{$\mathrm{NE}$} & \multirow[t]{2}{*}{$\mathrm{NC}$} & \multirow[t]{2}{*}{ Autores } \\
\hline & base 10 & base e & & & & & \\
\hline Itacaré, BA & 1,83 & 4,21 & 0,92 & 26 & 98 & 379 & Este trabalho \\
\hline Vale do Ribeira, SP & 1,86 & 4,28 & 0,90 & 200 & 114 & 2159 & Di Stasi et al. 2002 \\
\hline Barcarena, PA & 2,28 & 5,24 & 0,94 & 17 & 259 & 764 & Amorozo 2002 \\
\hline
\end{tabular}




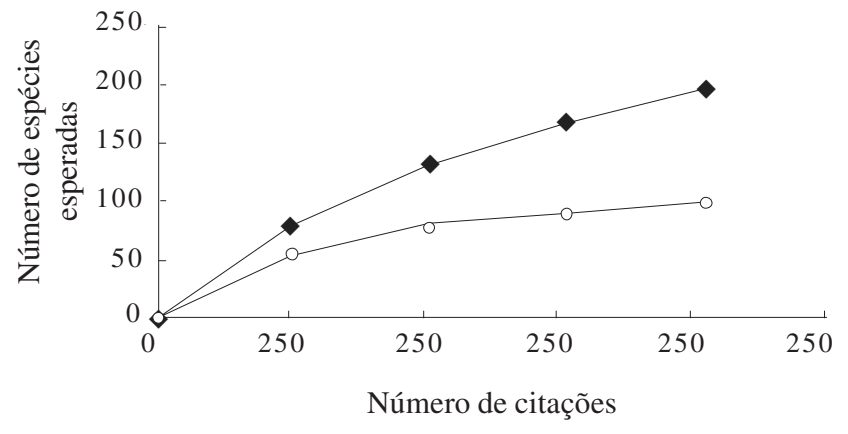

Figura 3. Curva de rarefação: número de citações $\times$ número de espécies esperadas em duas áreas estudadas. (-○- = Itacaré; $-\bullet-=$ Barcarena).

mulheres dominam melhor o conhecimento das plantas cultivadas próximas ao domicílio, isto é, no quintal ou jardim. Desta maneira, um esforço amostral mais concentrado no conhecimento popular que os homens detêm sobre plantas medicinais poderia resultar num maior número de espécies nativas medicinalmente usadas pelas comunidades rurais de Itacaré exatamento o que foi verificado em Barcarena, PA (Amorozo \& Gély 1988).

Algumas plantas medicinais são usadas juntas no mesmo preparado para, segundo os entrevistados, aumentar a eficácia do tratamento. Assim, é preparado, por exemplo, o lambedor (sinônimo de xarope, porém sua preparação varia um pouco de acordo com o informante; em geral, consiste em levar o chá de ervas, com outros ingredientes se desejável, para engrossar com açúcar ou rapadura no fogo) para o tratamento de gripe e de febre: juntam-se folhas de capim-santo (Cymbopogon citratus Stapf.), limão-merim (Citrus aurantiifolia Swingle.), bangui (Polygala cf. paniculata L.), fedegoso (Senna occidentalis (L.) Link.) e alho (Allium sativum L.). Em alguns casos, há restrições ou necessidade de cuidados durante um tratamento como, por exemplo: mulher grávida não pode tomar preparados feitos com plantas amargas; beber o sumo da graviola para picada de cobra sem dizer o nome da planta; não comer alimentos "pesados" ou "quentes", como carne de porco, pimenta, etc., quando se está fazendo tratamento com a goma da batata.

Já as "doenças culturais" apresentam simbologias interessantes. No benzimento, por exemplo, se a planta usada murchar, significa que a pessoa benzida estava com mau-olhado (muitos associam como causa, a inveja de outras pessoas, a qual gera um mal-estar, falta de ânimo ou fraqueza em quem está com mau-olhado).

Por um lado, o acesso facilitado à medicina alopática traz um benefício enorme para as comuni- dades no caso de doenças graves, fato inclusive reconhecido por eles. No entanto, percebe-se que muitas doenças que eram tratadas com as plantas existentes no próprio quintal do informante, atualmente são tratadas com remédios de farmácia. No sentido tradicional, isso traz dois prejuízos: a perda da prática do uso da medicina popular e um gasto financeiro muito alto com remédios. Outro aspecto problemático é a automedicação sem diagnóstico clínico, isto é, alguns informantes fazem tratamento com remédios indicados por terceiros. Talvez esta seja uma das causas da baixa frequiência de visita ao médico, aliada ao fato de, muitas vezes, não poderem pagar o tratamento necessário. Foram apontadas três principais razões que levam 38\% dos entrevistados a visitar com mais freqüência o médico: sentirem-se muito mal, não saberem a doença que têm ou o remédio caseiro não ter sido eficiente.

A origem do conhecimento destas comunidades em relação à utilização de plantas medicinais está ligada às pessoas mais idosas ( $58 \%$ dos casos), a outras pessoas, à utilização intuitiva das plantas, aos médicos ou aos livros. A grande maioria dos entrevistados (85\%) disse ter o costume de transmitir seus conhecimentos sobre a medicina popular a outras pessoas interessadas, porém apenas $27 \%$ disseram ter o costume de ensinar às pessoas mais jovens da própria família.

Em relação a percepção dos informantes sobre a área em que vivem, a maioria dos entrevistados vê as mudanças nela ocorridas de uns anos para cá de forma positiva. A chegada da Rodovia Itacaré-Ilhéus, uma das principais causadoras de impacto em toda a área, facilitou o acesso dos moradores às cidades próximas. A feira na cidade de Itacaré dinamizou o comércio de produtos da roça na cidade, ampliando alternativas de obtenção de renda. Outros fatores que contribuíram para a melhoria da vida dos moradores, segundo eles mesmos, foram: possibilidade de emprego na cidade e o turismo que permite a venda de produtos (como o coco, a tapioca, o abacaxi, etc.) na praia.

A maior dificuldade que a maioria dos entrevistados enfrenta é a de sobreviver sem poder "abrir roça" (isto é, não é permitido desmatar ou queimar novas áreas para plantio ou qualquer outro fim) depois que a área se tornou uma APA. Assim, $27 \%$ dos entrevistados não sabem apontar nenhum aspecto positivo gerado pela implantação da Unidade de Conservação, apesar de todos afirmarem o quanto é importante a conservação da mata.

Alternativas econômicas para o desenvolvimento sustentável destas comunidades chegaram mais tarde (através do projeto monitorado pela equipe do IESB) 
e, ainda não conseguiram abranger toda a população rural, como a comercialização de produtos orgânicos, produção de mudas nativas para reflorestamento, ecoturismo e implantação de sistemas agroflorestais. Dos 26 entrevistados, $62 \%$ participam do projeto. Entretanto, apenas $15 \%$ estão envolvidos com mais de uma destas atividades.

O contato cada vez maior entre os moradores destas comunidades rurais e pessoas vindas de fora é estimulado, principalmente, pelo turismo e pelo comércio de produtos da roça. O turismo atrai, além de visitantes, pessoas interessadas em empreendimentos como pousadas e restaurantes, nos quais acabam sempre empregando jovens nativos da zona rural. Essa ampla oportunidade de contato com pessoas vindas de outros lugares, com outros costumes, acaba por influenciar práticas locais, inclusive no cultivo e utilização de determinadas plantas. Além disso, a maior freqüência com que os moradores, em geral, passam a se deslocar até a cidade acaba tornando mais comuns as visitas ao médico e/ou o consumo em farmácias. Consequientemente, ocorre a redução das práticas medicinais caseiras e, algumas vezes, a perda da crença no poder de cura das plantas. Outro fator que pode estar reduzindo o uso da medicina popular (assim como a crença nesta) é a penetração da religião evangélica, a qual não permite certas práticas como, por exemplo, o benzimento.

Diante da situação sócio-econômica-ambiental das comunidades em questão, a conservação de suas práticas medicinais populares poderia ser estratégica. Afinal, o potencial de comercialização destes produtos medicinais caseiros (aqueles de eficácia farmacológica já comprovada cientificamente), devido ao assédio turístico crescente na região, levaria a mais uma alternativa econômica para a sustentabilidade destas comunidades e a valorização de seus conhecimentos e práticas populares.

A partir deste trabalho, foi feito o levantamento bibliográfico da eficácia farmacológica e eventuais cuidados na administração das espécies medicinais identificadas. Como retorno à comunidade, foram produzidas apostilas contendo tanto as informações obtidas durante o trabalho, isto é, o conhecimento etnobotânico das duas comunidades estudadas, como as observações necessárias pesquisadas posteriormente durante o levantamento bibliográfico. Estas apostilas foram apresentadas e distribuídas à equipe do IESB responsável pelos projetos desenvolvidos na área e, inicialmente, às 26 famílias que participaram da pesquisa.

\section{Agradecimentos}

À FAPESP, pela bolsa de Iniciação Científica (processo 01/09246-5); à equipe do IESB, pelo apoio logístico, sugestões e incentivo; às comunidades da Marambaia e Camboinha, pela troca de conhecimentos, amizade e grande sabedoria que eu tanto respeito e admiro; aos meus pais por todo carinho e motivação; à Maria Christina de Mello Amorozo e Antonio Furlan, pela tão significante orientação.

\section{Referências bibliográficas}

Almeida, C.F.C.B.R. \& Albuquerque, U.P. 2002. Uso e conservação de plantas e animais medicinais no Estado de Pernambuco (Nordeste do Brasil): um estudo de caso. Interciência 27(6): 276-285.

Amorozo, M.C.M. 1997. Algumas notas adicionais sobre o emprego de plantas e outros produtos com fins terapêuticos pela população cabocla do Município de Barcarena, PA. Brasil. Boletim do Museu Paraense Emilio Goeldi, Série Botânica, 13(2): 192-213 (reedição).

Amorozo, M.C.M. 2002. Uso e diversidade de plantas medicinais em Santo Antonio do Leverger, MT, Brasil. Acta Botanica Brasilica 16(2): 189-203.

Amorozo, M.C.M. \& Gély, A.L. 1988. Uso de plantas medicinais por caboclos do Baixo Amazonas. Boletim do Museu Paraense Emilio Goeldi, Série Botânica, 4(1): 47-131.

Área de Proteção Ambiental Itacaré/Serra Grande. 1998. Plano de manejo: zoneamento ecológico-econômico e plano de gestão. Salvador, BA.

Begossi, A. 1996. Use of ecological methods in ethnobotany: Diversity Indices. Economic Botany 50(3): 280-289.

Begossi, A.; Leitão Filho, H.F. \& Richerson, P.J. 1993. Plant uses in a brazilian coastal fishing community (Buzios Island). Journal of Ethnobiology 13(2): 233-256.

Bennett, B.C. \& Prance, G.T. 2000. Introduced plants in the indigenous pharmacopoeia of Northern South America. Economic Botany 54(1): 90-102.

Bernard, H.R. 1988. Research methods in cultural anthropology. Newbury Park, SAGE Publications.

Castellucci, S.; Lima, M.I.S.; Nordi, N. \& Marques, J.G.W. 2000. Plantas medicinais relatadas pela comunidade residente na Estação Ecológica de Jataí, Município de Luis Antonio/SP: uma abordagem etnobotânica. Revista Brasileira de Plantas Medicinais 3(1): 51-60.

Di Stasi, L.C.; Oliveira, G.P.; Carvalhaes, M.A.; QueirozJunior, M.; Tien, O.S.; Kakinami, S.H. \& Reis, M.S. 2002. Medicinal plants popularly used in the Brazilian Tropical Atlantic Forest. Fitoterapia 73: 69-91

Friedman, J.; Yaniv, Z.; Dafni, A. \& Palewitch, D. 1986. A preliminary classification of the healing potential of medicinal plants, based on a rational analysis of an ethnopharmacological field survey among bedouins in the negev desert, Israel. Journal of Ethnopharmacology 16: $275-287$. 
Hanazaki, N.; Tamashiro, J.Y.; Leitão Filho, H.F. \& Begossi, A. 2000. Diversity of plant uses in two Caiçara communities from the Atlantic Forest coast, Brazil. Biodiversity and Conservation 9: 597-615.

Krebs, C.J. 1989. Ecological Methodology. New York, Harper \& Row, Publ.

Lima, R.X.; Silva, S.M. \& Silva, Y.S.K.L.B. 2000. Etnobiologia de comunidades continentais da Área de Proteção Ambiental de Guaraqueçaba - Paraná - Brasil. Etnoecológica 4(1): 33-55.

Magurran, A.E. 1989. Diversidad ecológica y su medición. Espanha, Ediciones Vedrà.

Marodin, S.M. \& Baptista, L.R.M. 2002. Plantas medicinais do Município de Dom Pedro de Alcântara, Estado do Rio Grande do Sul, Brasil: espécies, famílias e usos em três grupos da população humana. Revista Brasileira de Plantas Medicinais 5(1): 1-9.

Martin, G. J. 1995. Ethnobotany: a methods manual. London, Chapman \& Hall.

Ming, L.C. 1996. Coleta de plantas medicinais. Pp. 69-86. In: L.C. Di Stasi (org.). Plantas medicinais: arte e ciência. São Paulo, Editora da Universidade Estadual Paulista.
Moerman, D.E. \& Estabrook, G.F. 2003. Native Americans' choice of species for medicinal use is dependent on plant family: confirmation with meta-significance analysis. Journal of Ethnopharmacology 87: 51-59.

Nolan, J.M. \& Robbins, M.C. 1999. Cultural conservation of medicinal plant use in the Ozarks. Human Organization 58(1): 67-72.

Organização Mundial da Saúde. 2000. CID - 10: Classificação Estatística Internacional de Doenças e Problemas Relacionados à Saúde. São Paulo, Editora da Universidade de São Paulo.

Silva Filho, S.R. 2000. Gestão participativa de recursos naturais e florestas - Construindo um modelo de desenvolvimento sustentável das comunidades rurais na Região de Itacaré - Serra Grande. Ilhéus, Instituto de Estudos Sócios-Ambientais do Sul da Bahia - IESB.

Valle, T.L. 2002. Coleta de germoplasma de plantas cultivadas. In: M.C.M. Amorozo; L.C. Ming \& S.P. Silva (eds.). Métodos de coleta e análise de dados em etnobiologia, etnoecologia e disciplinas correlatas. Pp. 129-154. In: Anais do I Seminário de Etnobiologia e Etnoecologia do Sudeste. Rio Claro, Coordenadoria de Área de Ciências Biológicas, Gabinete do Reitor, UNESP/ CNPq.

Voeks, R.A. 1996. Tropical forest healers and habitat preference. Economic Botany 50(4): 381-400. 